\title{
Instruments for the Navigation of Aircraft.
}

By G. M. B. Dobson.

THE design of instruments to aid the navigation of aircraft, like all other branches of aeronautics, has been greatly accelerated by the war, mediately becomes untrustworthy. It must be remembered that in a cloud the pilot of an aeroplane has no means of knowing whether he is turning to right or left, or flying straight. Thus previously the compass was used both to keep a straight course and to obtain the bearing of that course. The gyroscopic turn indicator-originally due to a suggestion by Prof. J. B. Hendersonis now available in several forms, all of which work well, and enable a pilot to turn and straighten out again while in a cloud almost as easily as in clear air. With the presence of this instrument the compass is required only for showing the direction of flight during the time the aeroplane is flying straight.

The gyroscopic turn indicator depends for its action on the precession of a rotating gyroscope against suitable controlling springs, when its axis

FIG. I.-R.A.E. gyro turn indicator Mark V. $a$, gyro wheel; $b, b$, oblique holes for air-drive; $c$, shaft carrying gyro wheel communicating motion to pointer; $d$, cam on shaft $c ; \varepsilon$, controlling sprisg; $f$, cam altering tension of sping $e$, worked by lever $g$.

though many of the instruments required are only now reaching their final stages of development, and instruments to effect navigation-as distinct from pilotage by the aid of landmarks--were scarcely used during the war.

Before and during the earlier stages of the war the errors of the magnetic compass as used on aeroplanes were the subject of much discussion, and numerous designs were tested with the view of overcoming its defects, which are, unfortunately, really inherent. The gyroscopic turn indicator has now solved this problem by taking over some of the original duties of the compass. Provided the aeroplane be flying

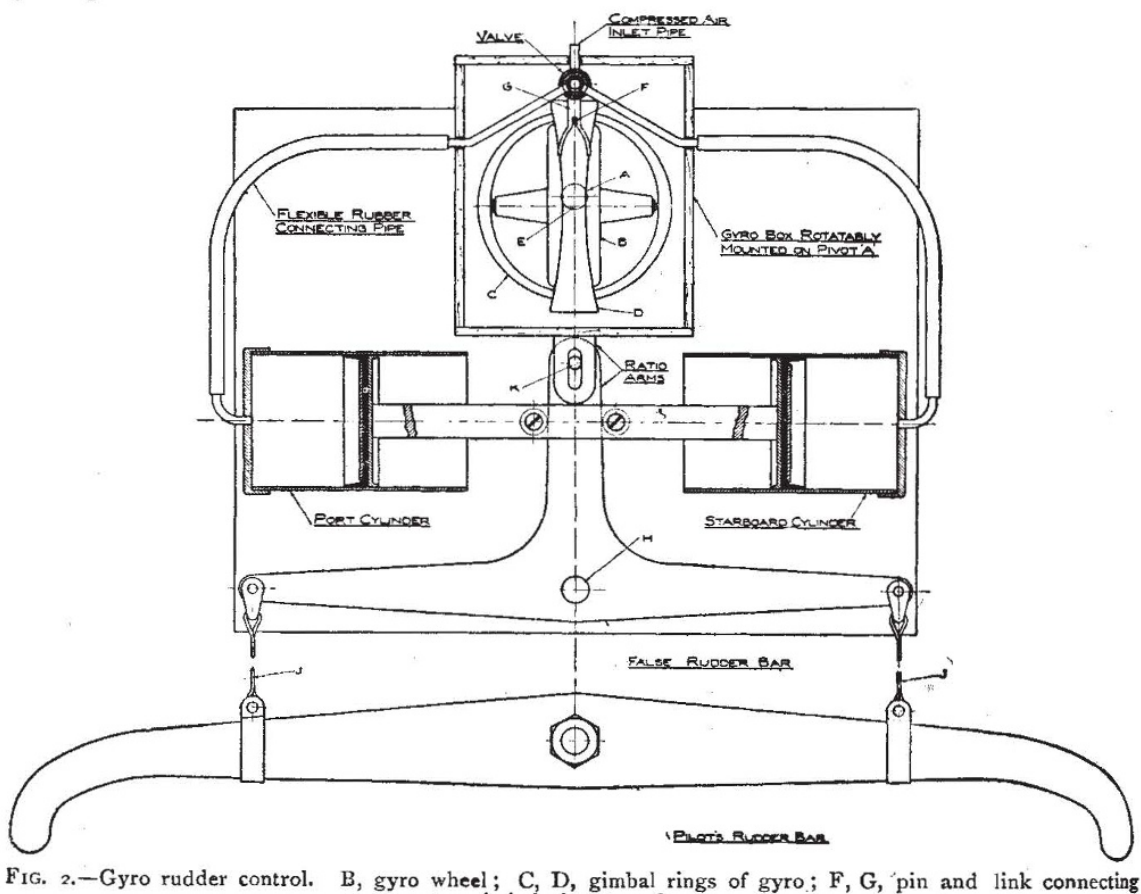

FIG. 2.-Gyro rudder control. B, gyro wheel; C, D, gimbal rings of gyro; F, G, pin and link connecting straight, the compass shows the direction of flight $\mid$ of rotation is changed. One simple form is shown correctly, but as soon as a turn is started it im- $\mid$ in Fig. I. The gyro wheel seen on the right is NO. 2668 , voL. I06] 
mounted outside the aeroplane, and spun at I0,000 to 15,000 revolutions a minute by the relative wind. The central cylindrical portion passes inwards through the side of the aeroplane and carries the dial, which is in front of the pilot. By means of a cam marked $f$ the tension of the control spring $e$ can be varied at will over a large range, and thus the sensitiveness of the instrument can be changed to suit different machines or different atmospheric conditions.

Another gyroscopic instrument which has been designed during and since the war is the gyro-
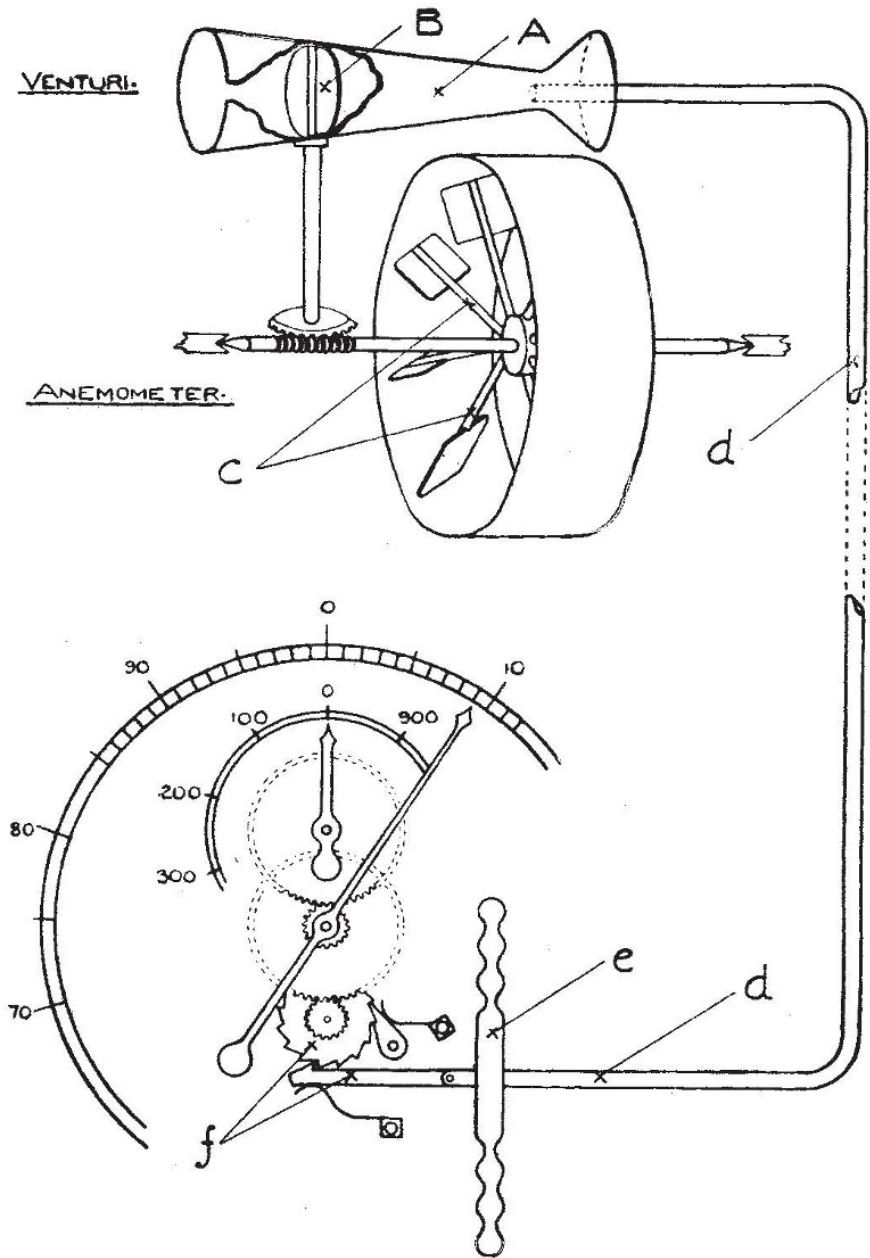

FIG. 3.-R.A.E. pneumatic air log. A, Venturi tube exposed to wind ; B, disc in exit cone revolved by wind nill $\mathrm{C} ; d$, pipe connecting throat of Venturi to dianbragm $c$ :

scopic rudder control. This is intended to relieve the strain on the pilot during a long flight by controlling the aeroplane's direction. With it the pilot need only check the course once every five to ten minutes. The instrument consists of a gyroscope hung freely in gimbals and carefully balanced. With good workmanship such a gyroscope will keep its direction within a degree in ten minutes. Any movement of the aeroplane relative to the gyroscope immediately operates a valve controlling a pneumatic servomotor, which puts over the rudder so as to turn the aeroplane NO. 2668. VOL. 106] back to its original course. It is also fitted with a simple "follow-up gear," so that the deflection of the rudder is proportional to the amount the aeroplane is off its true course. The apparatus is shown diagrammatically in Fig. 2. The gyroscopes are about 3 in. in diameter, and spun at about ${ } 5,000$ revolutions a minute by compressed air.

Until recently the only method of finding the distance an aeroplane had travelled through the air was to note the length of time flown and the average reading of the air-speed indicator. The latter reading, being dependent on the density of the air, must be corrected for the density at the height flown, as well as for a small error due to interference of the air-flow by the neighbouring parts of the aeroplane. To eliminate this trouble, an air $\log$ was designed, which should record continuously the actual distance travelled through the air. This is shown diagrammatically in Fig. 3. A windmill type of anemometer is employed, since this has the advantage that it needs no correction for change of density. As the windmill must necessarily be fixed on an exposed part of the aeroplane, it must be made to transmit its indications to the pilot. This is conveniently arranged by mounting a Venturi tube alongside the windmill. The exit of this Venturi tube is alternately opened and closed by a rotating disc, say once every Iooo revolutions of the windmill. The pressure at the throat of the Venturi tube is thus alternately above and below atmospheric pressure, and a pipe led from the Venturi tube to the indicator transmits these alternations of pressure, which operate the counting gear through the agency of a small diaphragm. The addition of a small adjustable baffle behind the windmill allows the interference error to be eliminated once for all for any aeroplane.

The use of ordinary sextants in the air is largely ruled out by the fact that the true horizon is nearly always obscured by haze. The top of this layer of haze is generally nearly horizontal, and has, therefore, sometimes been used instead of the real horizon. As, however, it may occasionally be inclined to the horizontal by a degree or more, such results are untrustworthy.

The use of bubble sextants has been attended by greater success than was at first expected. On aeroplanes the mean of six readings will generally give the altitude of the sun or star correctly within about ten or fifteen minutes of arc. On airships much better accuracy seems possible. The design of bubble sextants for use in the air is largely a matter of making them convenient to use. The image of the sun and bubble must move together across the field if the instrument be tilted, and the size of the bubble must be adjustable. The general 
principle of a bubble sextant is shown diagramNavigation of Aircraft," read to the Royal Geomatically in Fig. 4. By means of a lens and prism graphical Society by the author on May ro last, (not shown), which :are fixed above the bubble lens, the horizon can also be seen in the field of view, as well as the bubble, so that the instrument may be used as an horizon sextant if required.

The problem of the rapid calculation of sextant observations appears to have been solved completely by the cylindrical slide-rule due to $\mathrm{Mr}$. Bygrave. With a sliderule about 7 in. long results can be obtained in three or four minutes' time which are accurate to within about $\mathrm{three}$ minutes of arc. With larger patterns greater accuracy in obtained.

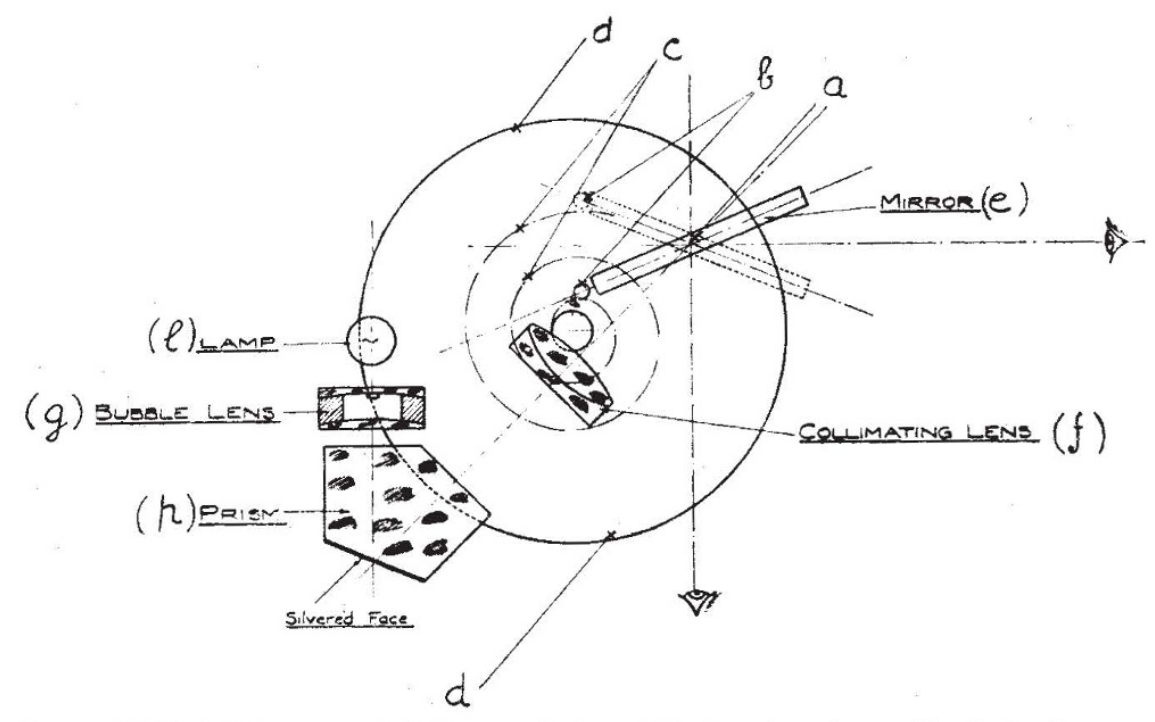

FIG. 4.-R.A.E. bubble sextant Mark II. $a$, axis about which clear mirror $e$ is moved by pin $\delta$ resting on cam $c$ fixed to drum $d$. Image of bubble in lens cell $g$ seen by reflection in $e$ through lens $f$ after further reflection in prism $p$. Bubble illuminated at night by lamp $l$.

The accompanying illustrations are from a and published in the Geographical Journal for lecture on "The Design of Instruments for the November.

\section{Industrial Research Associations.}

\section{VI.-The Glass Research Association. \\ By EDWARd Quine.}

THE Glass Research Association has been estab1 lished for investigation into the problems of the glass industry in accordance with the scheme of the Committee of the Privy Council for Scientific and Industrial Research. The association received its Certificate of Incorporation on August II, I919, and held the first general meeting on October I4 following.

The objects of the association are to conduct scientific and technical investigations relating to glass and its manufacture, and to disseminate among members technical and other information bearing on these subjects and on the production of articles made wholly or partly of glass.

The rate of development of the glass industry in this country is largely influenced by difficulties in the matter of technique, works organisation, production and equipment, and, in order that the industry may attain a high degree of efficiency, it is necessary for investigations to be directed towards overcoming these difficulties, in addition to investigations of fundamental principles and search for new knowledge. To ensure that greater economy in production and more satisfactory products may be obtained, improved methods must be introduced, and the works practice of the industry brought into line with the advanced methods found operative in other countries. Until recent years no comprehensive research work reNo. 2668 , vol. I06] lating to the industry had been carried out in this country, but the need for a deeper and more extensive knowledge of the fundamental facts underlying the various processes of manufacture is now appreciated. The association is consequently endeavouring to secure that the fundamental principles and their application shall be thoroughly investigated by systematically conducted researches, so that, side by side with the modernising of works practice, and the introduction into the industry of the scientific control of the various operations, new knowledge may be acquired which may ultimately lead to industrially valuable developments.

The investigations of the association cover the problems of all sections of the glass industry other than those of optical glass, research work in which is being undertaken by the British Scientific Instrument Research Association.

The membership of the association is limited to British corporations and British subjects carrying on business in connection with the manufacture of glass, and other trades and industries allied therewith or accessory thereto. Individuals ineligible for membership or not desiring admission as members, who are interested in the glass industry and willing to subscribe to the objects of the association, may be admitted by the council as 